\title{
Balancing Uplink and Downlink Delay of VoIP Traffic in WLANs using Adaptive Priority Control (APC)
}

\author{
Sangho Shin, Henning Schulzrinne \\ Columbia University Dept of Computer Science \\ Email: $\{$ ss2020,hgs $\}$ cs.columbia.edu
}

\begin{abstract}
In IEEE 802.11 wireless networks, the downlink delay rises as the number of VoIP nodes increases while the uplink delay remains small due to the same chance of media access between nodes and the Access Point (AP). This degrades the capacity and QoS of VoIP significantly. Therefore, we introduce Adaptive Priority Control (APC) to balance the downlink and uplink delay of VoIP traffic at the MAC layer, by giving to the AP a higher transmission priority, which is adaptively decided according to the uplink and downlink traffic volume. And, we verify through theoretical analysis that $A P C$ is an optimal method to balance the uplink and downlink delay.
\end{abstract}

\section{INTRODUCTION}

With VoIP replacing current circuit-switched PBX (Private Branch eXchange), the number of wireless VoIP users is increasing. However, the Quality of Service (QoS) of VoIP in current IEEE 802.11 wireless networks cannot match the growth of the use of VoIP in wireless networks. In current IEEE 802.11 wireless networks, as the number of VoIP flows increases, the downlink delay increases while the uplink delay stays very low according to our simulations (Fig. 1 shows the results of our simulation in IEEE $802.11 \mathrm{~b}$. The parameters used are shown in Section V). The reason is that while the AP and nodes have the same chance to send packets in the current default Multiple Access Control (MAC), Distributed Coordination Function (DCF), the AP typically needs to send more packets than each node. In VoIP traffic, uplink and downlink traffic volume is roughly the same, and for better performance, both delay components should be balanced.

In this paper, we introduce Adaptive Priority Control (APC), which adaptively balances the uplink and downlink delay while increasing the capacity for VoIP by $25 \%$. APC computes an optimal priority of the AP for balancing the two components according to the uplink and downlink VoIP traffic volume. In particular, we use the contention free transmission method in controlling transmission rate, in which the AP transmits multiple frames in a row without backoff, while many other approaches [1] [2][3] use the contention window (CW) size control approach, which increases the collision rate of frames and decreases the channel utilization, as we will show in Section V-C. Furthermore, APC requires modifications only in the AP, and it is completely compatible with clients that use legacy 802.11 cards. We also believe that APC can be implemented easily with IEEE 802.11e [4] or Wireless

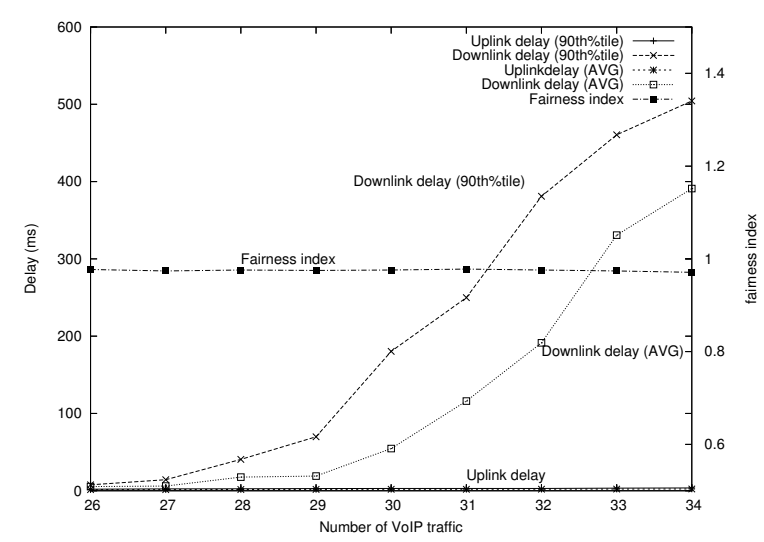

Fig. 1. The Delay of VoIP traffic in DCF

Media Extension (WME) feature which is supported in many commercial wireless cards.

This paper is organized as follows: In Section II, we present some related study regarding to the fairness issues in VoIP; In Section III, we introduce our algorithm; in Section IV, we analyze APC theoretically; in Section V, we show the simulation results of APC in various scenarios; in Section VI, we describe how to implement APC practically.

\section{RELATED WORK}

Many papers have studied fairness among wireless nodes ([1] [2] [3] [5]), and some papers have also considered the fairness between the AP and wireless nodes ([6], [7]). However, they have focused only on throughput fairness and failed to consider the balance of the end-to-end delay, which is more important in VoIP traffic. As shown in Fig. 1, the Jain's Fairness Index [8], which is generally 1 when every node shares the throughput equally and was computed including all wireless and Ethernet nodes, is very close to 1 even when uplink and downlink delay are significantly unbalanced.

The following papers considered the balance of uplink and downlink delay. Wang et al. [9] introduced the New Fair MAC to improve the fairness and delay of VoIP traffic. When a station wins a transmission opportunity, it is allowed to transmit a burst of packets instead of a packet. However, allowing stations to transmit a burst of packets does not help much for VoIP traffic because only one VoIP packet is sent 
every packetization interval. Also, for fairness between stations, Wang et al. introduces Max Transmission Time (MTT). Considering the packetization intervals are usually $10 \mathrm{~ms}$ to $40 \mathrm{~ms}$ and the uplink delay is very low even when the number of VoIP nodes exceeds the capacity (Fig. 1), only one packet will be sent during the MTT as in DCF, and for this reason, the delay decreased by only a few milliseconds. Casetti et al. [10] improved the fairness for VoIP between nodes and the AP in IEEE 802.11e Enhanced Distributed Channel Access (EDCA) by differentiating frames based on traffic type and also direction. They found the optimal Contention Window (CW) values for the best fairness and throughput of VoIP traffic via simulation, and improved the capacity of VoIP by around $15 \%$. However, they tested the optimal $\mathrm{CW}$ values with only one type of VoIP traffic, and failed to show that the optimal value works for other types. In our study, it was found that the optimal parameters should be changed according to the number of VoIP nodes and type of VoIP traffic. Also, we found that changing $\mathrm{CW}$ values to control the priority of frames has limitations, which will be shown in Section V-C. In our approach, the parameter for fairness changes adaptively according to the network condition and we use contention free transmission approach, which does not have such limitations.

\section{AdAPtive Priority CONTROL (APC)}

As we mentioned in the introduction, the uplink and downlink delay need to be balanced for better QoS and capacity. Both uplink and downlink delay are dominated by the queuing delay when the channel is very congested, considering that the transmission and propagation delay in IEEE 802.11 wireless networks are very small relatively to the queuing delay. In particular, the queue size of the AP is much larger than that of wireless nodes when there are a large number of VoIP sources because the AP receives all packets to all the wireless nodes, thus the queuing delay is also much bigger than the one of wireless nodes. Therefore, the AP needs to have more chance to transmit frames in order to balance the uplink and the downlink delay. In this paper, we propose APC which differentiates the priority of the AP from that of wireless nodes adaptively according to wireless channel condition and the uplink and downlink traffic volume.

Before we mention how to decide the optimal priority of the AP to balance the uplink and downlink delay, we discuss how to apply the priority of the AP at the MAC layer. This is because the methods to apply the priority usually cause overhead and the overhead affects the priority decision algorithm.

In IEEE 802.11, there are three well-known methods to control the priority of wireless nodes. All three methods are used in IEEE 802.11e in order to differentiate the priorities of frames according to the Access Category (AC). The first method is to control contention window (CW) size. The backoff time of a frame is chosen randomly between 0 and $\mathrm{CW}$ value. When nodes have a smaller window size, the backoff time becomes smaller and the transmission rate becomes higher. The problems of this method, however, are

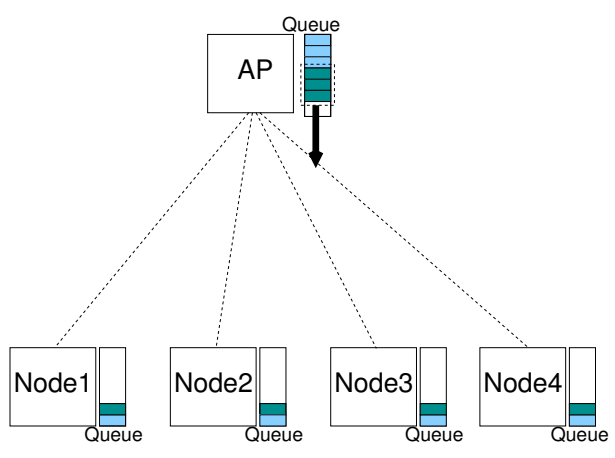

Fig. 2. Packet transmission in APC

that the collision rate increases as the window size decreases [11], and that it is difficult to accurately control the priority since the backoff time is chosen randomly within CW size. In particular, the first one is a big overhead we wished to avoid. We will show our experiment results for it in Section $\mathrm{V}$-C. The second method is to change the Inter-Frame Spacing (IFS). A smaller IFS causes the backoff time to decrease faster and the transmission rate to increase naturally, and the node with the smaller IFS wins the channel when two nodes try to transmit frames at the same time. However, we cannot control accurately the transmission rate using this method because the backoff time is still decided randomly, as in the first method.

The last method is to transmit multiple frames contention free (without backoff) when a node gets a chance to transmit a frame, and control the number of frames sent contention free, and this is called Contention Free Burst (CFB) in IEEE 802.11e. APC uses CFB because it allows us to control the transmission rate precisely according to the priority without overhead. Every node including the AP has the same chance to transmit frames in average in IEEE 802.11 [12]. Thus, if the AP sends $P$ frames contention free when it gets a chance to transmit a frame, then the AP gets exactly $P$ times higher priority than other wireless nodes.

For fairness between the downlink (the AP) and uplink (wireless nodes) in a Basic Service Set (BSS), when uplink and downlink have the same amount of traffic, the AP and the wireless nodes need to be able to send the same number of packets within a given interval. Then, intuitively, the AP needs to send $N$ frames while $N$ wireless nodes transmit a frame each (We call this 'semi-adaptive method' in this paper since it is adaptive to the change in the number of the active wireless nodes, but not to the change in the uplink and downlink traffic volume). In VoIP traffic, when a single packetization interval is used for all VoIP traffic in a BSS, the uplink and downlink traffic volumes are symmetric, in general with large number of VoIP sources, thus we can apply the semi-adaptive method to balance the uplink and downlink delay. However, when more than one packetization interval is used for VoIP in a BSS, the traffic volume of the uplink and downlink becomes asymmetric: even when the number of active wireless nodes and Ethernet nodes are the same, the number of packets from the wireless nodes and the Ethernet nodes depends on the 
packetization intervals of the active nodes. For example, when ten Ethernet nodes with $10 \mathrm{~ms}$ packetization interval and ten wireless nodes with $20 \mathrm{~ms}$ packetization interval are talking with the same $64 \mathrm{~kb} / \mathrm{s}$ voice bit rate, the volume of the downlink traffic from Ethernet nodes is larger than the uplink traffic volume because of the overhead such as packet headers, even if the total voice data size is the same. In such a case, we need to consider the traffic volume of uplink and downlink in deciding the priority of the AP.

We propose to use the ratio of the number of packets in the queue of the AP and an average queue size of all wireless nodes as the priority of the $\mathrm{AP}(P)$ when the queue of wireless nodes is not empty, and the number of active wireless nodes when the queue is empty. That is, $P$ is calculated as follows:

$$
P= \begin{cases}\left\lceil\frac{Q_{A P}}{Q_{N o d e}}\right\rceil & \text { if } Q_{\text {Node }} \geq 1 \\ N_{e} & \text { if } Q_{\text {Node }}=0\end{cases}
$$

where, $Q_{A P}$ is the queue size of the AP, $Q_{\text {Node }}$ is the average queue size of the wireless nodes, and $N_{e}$ is the number of active Ethernet nodes.

For instance (Fig. 2), if four wireless nodes, Node 1, Node 2 , Node 3 and Node 4 have two packets in each queue, and the AP has six packets in the queue. Then, the average queue size of the wireless nodes is 2 , and the priority of the AP becomes three $(=6 / 2)$. Thus, the AP sends three frames contention free when it gets a chance to transmit a frame. If we assume that every node got the same chance to transmit frame(s), then the average number of packets in the queue of the wireless nodes and the one of the AP becomes one and three, respectively, and both of them become zero after the next transmission. Therefore, transmitting $Q_{A P} / Q_{N o d e}$ packets contention free results in the same packet processing time in the AP and wireless nodes, which means that the AP and wireless nodes have the same queuing delay. We will prove this theoretically with more general case in the next section. Also, in this way, the priority of the AP changes adaptively when the traffic volume of the uplink and downlink changes. When the amount of traffic to the AP caused by Ethernet nodes increases, the queue size of the AP increases and the priority also increases to balance the downlink delay with the uplink delay. When the queue size of the nodes increases, the priority of the AP decreases.

\section{THEORETICAL ANALYSIS}

In this section, we show that the AP needs to transmit $Q_{A P} / Q_{N o d e}$ packets when $Q_{N o d e}>1$ and $N_{e}$ packets when $Q_{\text {Node }}=0$ to balance the uplink and downlink delay.

We define the symbols used in the analysis as follows: $\Delta Q_{A P}=$ Change of the number of packets in the queue of the AP

$Q_{A P}=$ Number of packets in the queue of the AP

$Q_{N o d e}=$ Average number of packets in the queue of all wireless nodes

$D_{A P}=$ Queuing delay of the AP

$D_{\text {Node }}=$ Queuing delay of a node
$N_{e}=$ Number of active (talking) wired nodes

$x_{A P}=$ Transmission overhead (backoff, defer and retry) at the AP

$i=$ Packetization interval

$t=$ Transmission time of one VoIP frame including ACK

$\lambda=$ Packet arrival rate

$\mu=$ Packet transmission rate

$P=$ Priority of the AP to balance the uplink and downlink delay

The dominant component of delay is the queuing delay considering that the transmission delay and the transmission overhead are very small. Furthermore, the transmission delay is the same in the AP and wireless nodes assuming that they use the same transmission rate, and the transmission overhead, which includes backoff, defer and retransmission overhead is also similar for the AP and wireless nodes, while the queuing delay of the AP is much bigger than the one of the wireless nodes. Therefore, balancing the queuing delay of the AP and wireless nodes results in the balanced uplink and downlink delay. Thus, we show that APC balances the queuing delay of the AP and wireless nodes.

We can compute the queuing delay by multiplying the transmission time to the queue size according to Little's law $\left(D_{\text {system }}=Q_{\text {system }} / \mu_{\text {system }}\right)$. Without borrowing the law, we can easily infer that the queuing delay can be computed by multiplying the queue size by the transmission rate.

Then, we can compute the queuing delay of the AP $\left(D_{A P}\right)$ and the nodes $\left(D_{\text {Node }}\right)$ as follows:

$$
\begin{gathered}
D_{A P}=Q_{A P} \cdot \frac{1}{\mu_{A P}} \\
D_{\text {Node }}=Q_{\text {Node }} \cdot \frac{1}{\mu_{\text {Node }}}
\end{gathered}
$$

And, we consider the priority of the AP $(P)$ in two cases: When the queue size of nodes is greater than zero $\left(Q_{\text {Nodes }} \geq\right.$ $1)$ and when the queue size of nodes is zero $\left(Q_{\text {Nodes }}=0\right)$.

\section{A. When $Q_{\text {Nodes }} \geq 1$}

When all wireless nodes including the AP have packets to transmit, every wireless node as well as the AP has the same chance to transmit packets due to the fairness of CSMA/CA in average, that is, $\mu_{A P}=\mu_{N o d e}$, in DCF. Then, in APC, $\mu_{A P}=P \cdot \mu_{N o d e}$ because the AP transmits $P$ packets when it gets a chance to transmit packets while each node transmits only one packet. Thus, $D_{A P}$ can be rewritten as:

$$
D_{A P}=Q_{A P} \cdot \frac{1}{P \cdot \mu_{N o d e}}
$$

Then, we can get the optimal $P$ value for balancing the delay of wireless nodes and the AP as follows:

$$
\begin{gathered}
D_{A P}=D_{\text {Node }} \\
Q_{A P} \cdot \frac{1}{P \cdot \mu_{\text {Node }}}=Q_{\text {Node }} \cdot \frac{1}{\mu_{\text {Node }}}
\end{gathered}
$$




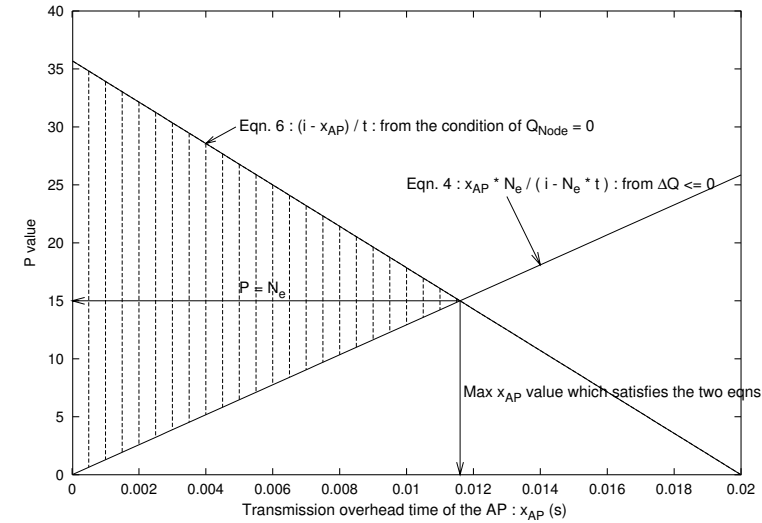

Fig. 3. Optimal $\mathrm{P}$ value when $Q_{\text {Node }}=0$

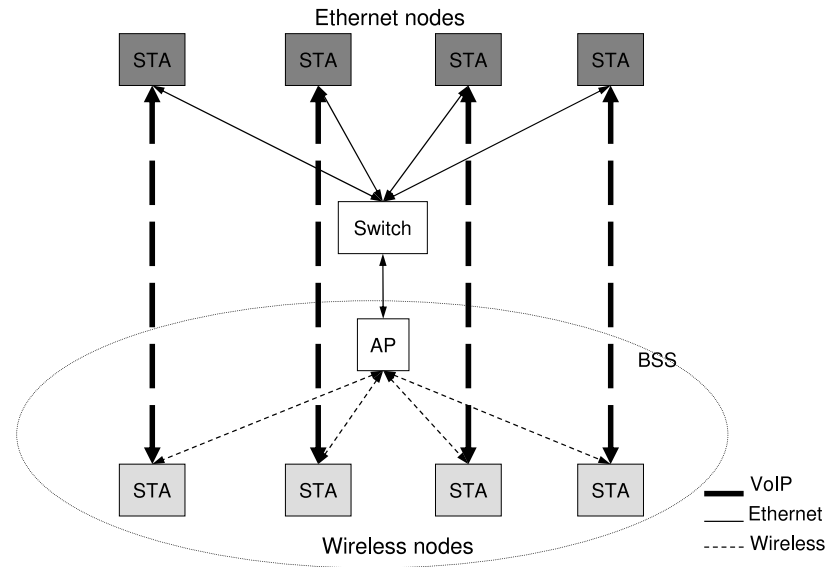

Fig. 4. Simulation topology

their queues empty. That is,

$$
x_{A P}+t \cdot P<i
$$

Then,

$$
P<\frac{i-x_{A P}}{t}
$$

Eqns 4 and 6 are plotted in Fig. 3 with $t=0.00056 \mathrm{~s}^{1}$, $N_{e}=15$ and $i=0.02 \mathrm{~s}$, and the shaded region represents the one that satisfies Eqn. 4 and Eqn. 6. According to the two graphs in Fig. 3, we can see that $P$ should be less than or equal to $N_{e}$. We can also get the same result when we combine Eqn. 2 and Eqn. 5:

$$
\begin{gathered}
\frac{N_{e}}{i}=\frac{P}{x_{A P}+t \cdot P} \geq \frac{P}{i} \\
P \leq N_{e}
\end{gathered}
$$

Therefore, the optimal $P$ value that satisfies the two equations in any possible $x_{A P}$ value is $N_{e}$.

\section{Simulation And RESUlts}

In order to evaluate the performance of APC, we have implemented APC and the semi-adaptive method in the QualNet simulator [13] and measured the uplink and downlink delay with various packetization intervals.

\section{A. Simulation parameters}

As shown in Fig. 4, we used the Ethernet-to-wireless network topology to focus on the delay in a BSS. In the simulations, the Ethernet portion added $1 \mathrm{~ms}$ of transmission delay, which allows us to to assume that the end-to-end delay is essentially the same as the wireless transmission delay.

We used IEEE 802.11b [14] and the parameters are shown in Table I. VoIP packets are encoded using G.711 codec with payloads of 80,160 , and 320 bytes, which represent packetization intervals of 10, 20 and $40 \mathrm{~ms}$, respectively (voice bit rate of $64 \mathrm{~kb} / \mathrm{s}$ ). We added an additional 12 bytes to the

\footnotetext{
${ }^{1}$ The $t$ value is calculated with $160 \mathrm{~B}(20 \mathrm{~ms}$ packetization interval and G.711 codec) payload in $11 \mathrm{Mb} / \mathrm{s}$ transmission rate
} 
TABLE I

PARAMETERS IN IEEE $802.11 \mathrm{~B}(11 \mathrm{MB} / \mathrm{s})$

\begin{tabular}{|l|r|}
\hline Parameters & value \\
\hline PLCP ${ }^{2}$ Preamble & $144.00 \mu \mathrm{s}$ \\
PLCP Header & $48.00 \mu \mathrm{s}$ \\
PLCP Header Service & $192.40 \mu \mathrm{s}$ \\
MAC Header+CRC & $36 \mathrm{~B}$ \\
RTS threshold & $1500 \mathrm{~B}$ \\
Retransmission limit & 7 frame \\
SIFS & $10 \mu \mathrm{s}$ \\
DIFS & $50 \mu \mathrm{s}$ \\
Slot & $20 \mu \mathrm{s}$ \\
$C W_{M I N}$ & 31 slots \\
\hline
\end{tabular}

TABLE II

VOICE PATTERN IN ITU-T P.59 (TEMPORAL PARAMETERS IN CONVERSATIONAL SPEECH)

\begin{tabular}{|l|r|r|}
\hline Parameter & Duration (s) & Fraction (\%) \\
\hline Talkspurt & 1.004 & 38.53 \\
\hline Pause & 1.587 & 61.47 \\
\hline Double Talk & 0.228 & 6.59 \\
\hline Mutual Silence & 0.508 & 22.48 \\
\hline
\end{tabular}

payload reflecting the overhead incurred by RTP [15] header. The VoIP packets were transported with UDP. We considered VoIP traffic with silence suppression, using the conversational speech model with double talk described in ITU-T P.59 [16]. The parameters are shown in Table II and the conversation model is shown in Fig. 5.

\section{B. Capacity for VoIP traffic}

The one-way end-to-end delay of voice packets should be less than $150 \mathrm{~ms}$ [17] [18]. We assumed the codec delay to be about $30-40 \mathrm{~ms}$ at both the sender and the receiver, and the backbone network delay to be about $20 \mathrm{~ms}$. Therefore, the wireless network should contribute less than about $60 \mathrm{~ms}$ to the total end-to-end delay.

We measured the 90th percentile value ${ }^{3}$ of the uplink and

${ }^{2}$ Physical Layer Convergence Protocol

${ }^{3}$ Generally, 90th percentile value is used for measuring QoS of VoIP because it indicates the jitter of VoIP applications.

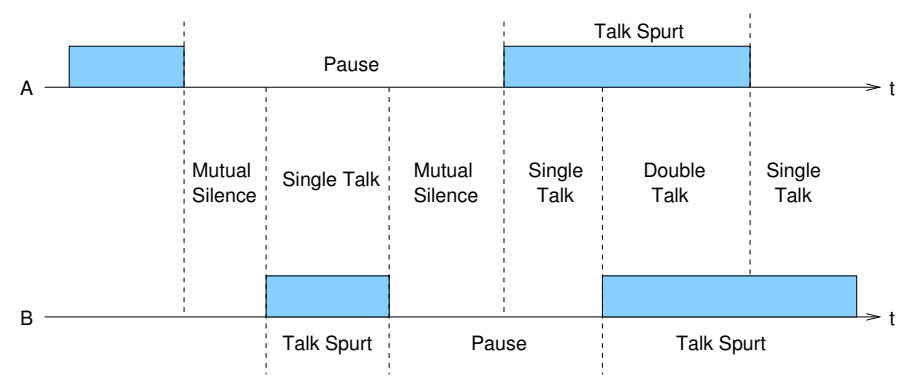

Fig. 5. Conversational speech model in ITU-T P.59 downlink delay of voice packets, and defined the capacity of VoIP as the maximum number of wireless nodes so that the average of the 90th percentile of the one-way end-to-end delay for both direction does not exceed $60 \mathrm{~ms}$.

\section{Simulation results}

1) Evaluation of APC and comparison with the semiadaptive method: Fig. 6 shows the simulation results with $20 \mathrm{~ms}$ packetization interval and $64 \mathrm{~kb} / \mathrm{s}$ VoIP traffic. We plotted both the 90th percentile and average value of uplink and downlink delay because the 90th percentile value is a good measure of the capacity for the VoIP traffic, and the average value is used to check the balance of the uplink and downlink delay. Fig. 6 shows both the semi-adaptive method and APC balance the uplink and downlink delay effectively when a packetization interval is used for all VoIP sources. And if we compare with the result of DCF (Fig. 1), we can see that APC improves not only the balance between uplink and downlink delay but also the capacity for the VoIP traffic by $25 \%$, from 28 calls to 35 calls.

Fig. 7 shows the simulation results when $10 \mathrm{~ms}$ and $20 \mathrm{~ms}$ packetization interval are used on both Ethernet and wireless nodes half and half. We can see that the uplink and downlink delay are unbalanced in the semi-adaptive method as the number of VoIP sources increases, while the two components are still balanced in APC. This is because when more than one packetization interval is used, the traffic volume of uplink and downlink becomes more asymmetric, and APC changes the priority of the AP adaptively to the change of the uplink and downlink traffic volume, while the semi-adaptive method is adaptive only to the change of the number of active wireless nodes.

We also evaluated the performance of APC with other packetization intervals, which decide the VoIP packet size and affect the capacity for VoIP traffic. Fig. 8 shows the simulation results of APC using the VoIP traffic with $40 \mathrm{~ms}$ packetization interval only (Fig. 8(a)) and with $20 \mathrm{~ms}$ and $40 \mathrm{~ms}$ packetization intervals half and half (Fig. 8(b)). It confirms that APC works in various types of VoIP traffic.

In order to see how the uplink and downlink delays change with simulation time, we have plotted the two components throughout the simulation time, and we have confirmed that uplink and downlink delay are balanced throughout the whole simulation. Fig. 9 shows a sample simulation result with 36 VoIP sources $(64 \mathrm{~kb} / \mathrm{s}$ and $20 \mathrm{~ms}$ packetization interval).

2) Comparison with $\mathrm{CW}$ control method: Fig. 10 shows the results using $\mathrm{CW}$ in controlling transmission rate. The priority of the AP was calculated using the same algorithm as in APC (Eqn. 1 in Section III), and the priority $(P)$ was converted to the transmission of the AP as follows.

$$
C W=\max \left(C W_{M I N} / P, 1\right)
$$

where, $P \geq 1$ from Eqn. $1,1 \leq C W \leq C W_{M I N}$, and the $C W_{M I N}$ is the minimum contention window size defined in IEEE 802.11. When the priority of the AP is very high $(P \geq$ $\left.C W_{M I N}\right), \mathrm{CW}$ decreases to 1 , then the AP transmits packets 


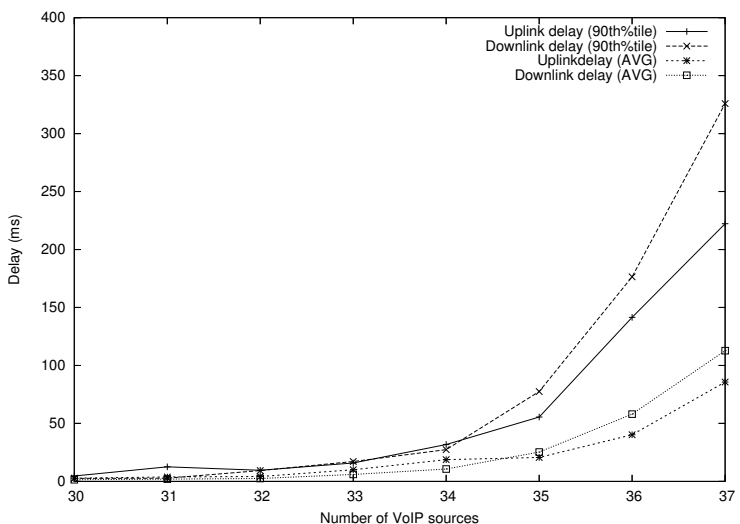

(a) The semi-adaptive method

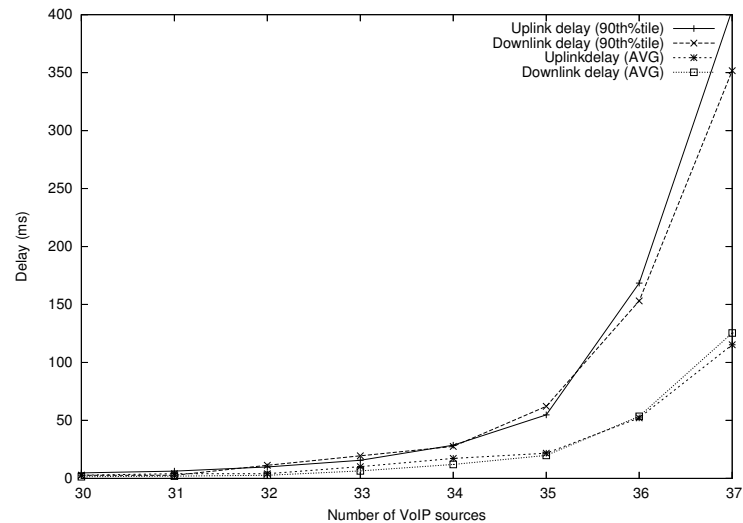

(b) APC

Fig. 6. Simulation results using two priority control methods with $20 \mathrm{~ms}$ packetization interval $64 \mathrm{~kb} / \mathrm{s}$ VoIP traffic

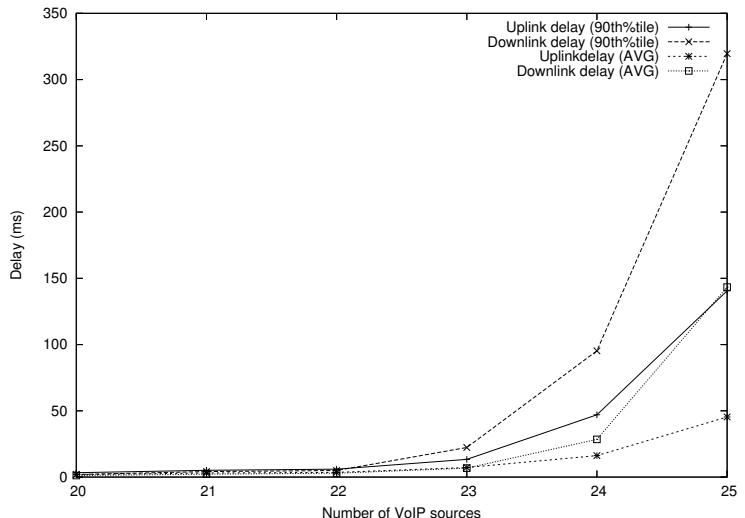

(a) The semi-adaptive method

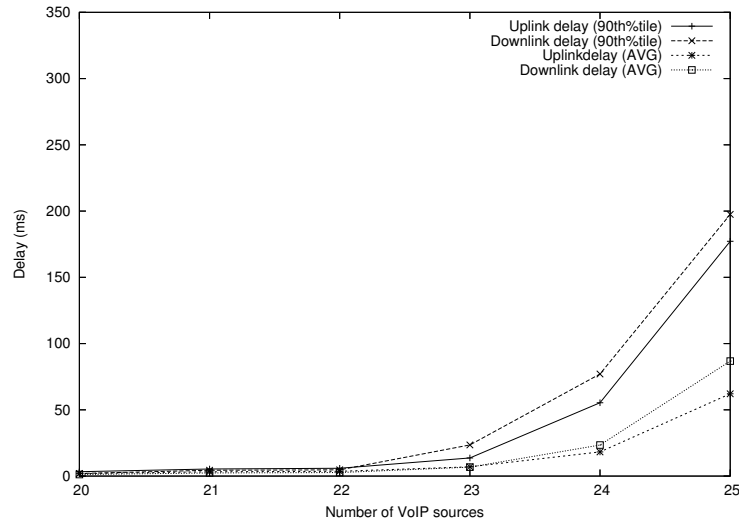

(b) APC

Fig. 7. Simulation results using two priority control methods with mixed $(10 \mathrm{~ms}$ and $20 \mathrm{~ms})$ packetization interval $64 \mathrm{~kb} / \mathrm{s}$ VoIP traffic

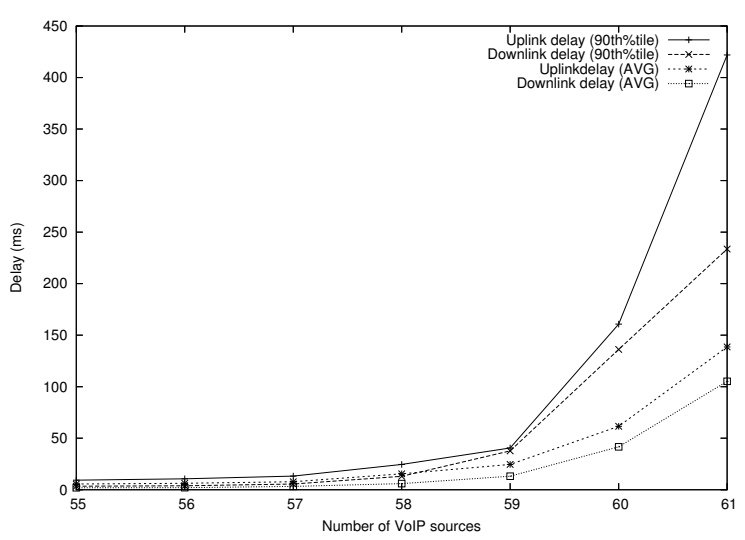

(a) $40 \mathrm{~ms}$ packetization interval $64 \mathrm{~kb} / \mathrm{s}$ VoIP traffic

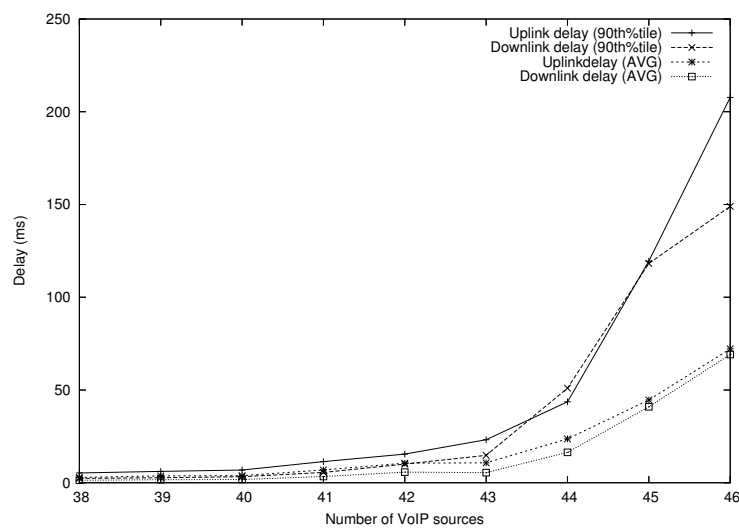

(b) $20 \mathrm{~ms}$ and $40 \mathrm{~ms}$ mixed packetization interval $64 \mathrm{~kb} / \mathrm{s}$ VoIP traffic

Fig. 8. Simulation results of APC using various packetization interval 


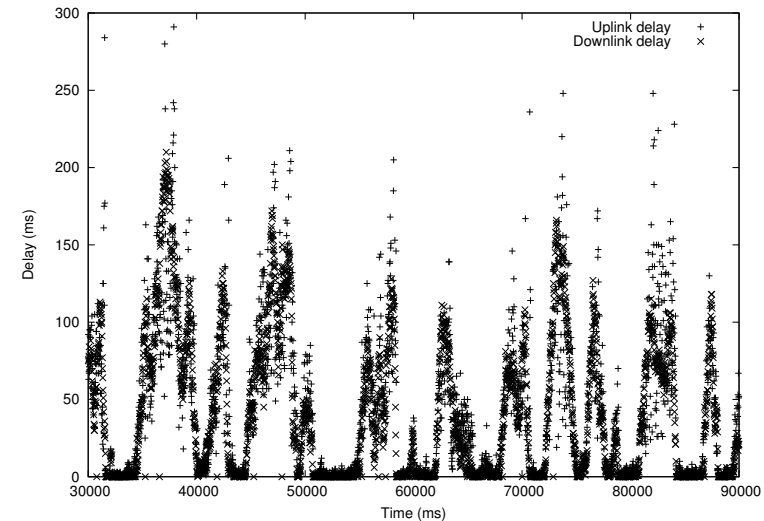

Fig. 9. The uplink and downlink delay with 36 VoIP sources using APC

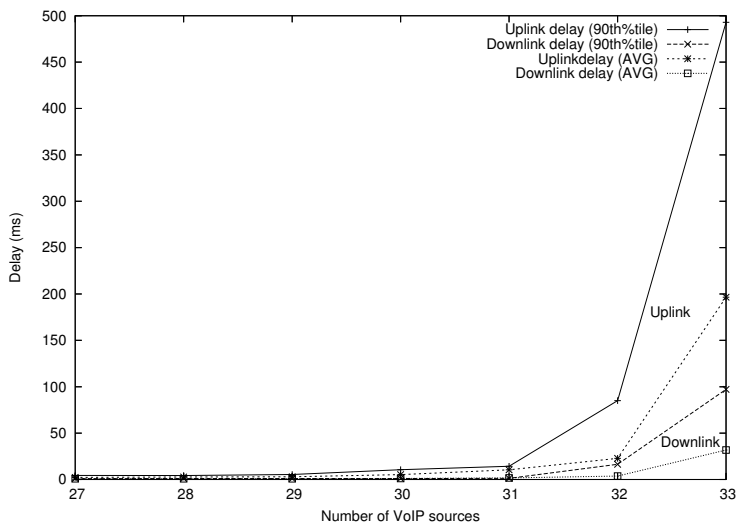

Fig. 10. Simulation results using $\mathrm{CW}$ in controlling transmission rate

almost without the backoff. When the priority is 1 , the CW becomes $C W_{M I N}$ and the $\mathrm{AP}$ has the same transmission rate with wireless nodes. As shown in Fig. 10, although the balance was a little bit improved compared to the case of DCF (Fig. 1), the uplink delay was much bigger than the downlink delay, which means that the AP was given too high priority. The reason is that even if the $\mathrm{CW}$ of the $\mathrm{AP}$ is changed to $1 / P$ of $C W_{M I N}$, the transmission rate of the AP is not exactly $P$ times because the backoff time is chosen randomly within the CW size. Another problem of this approach is the high retry rate, as shown in Fig. 11. We can see that the retry rate of the AP in $\mathrm{CW}$ control approach increases significantly as the number of VoIP sources increases, while the one in APC keeps the same retry rate. This is because a smaller CW causes higher collision in CSMA/CD with many wireless nodes. The reason why the retry rate of the AP in APC is lower than that in DCF is that contention free transmission of the AP decreases the probability of packet collision.

\section{IMPLEMENTATION ISSUES}

In order to use the ratio of the queue size of the AP and a node as the priority value of the AP, the AP needs to know the queue size of all wireless nodes as well as the queue size of itself. One way to implement it is that all the wireless nodes put their queue size to VoIP packets, and the

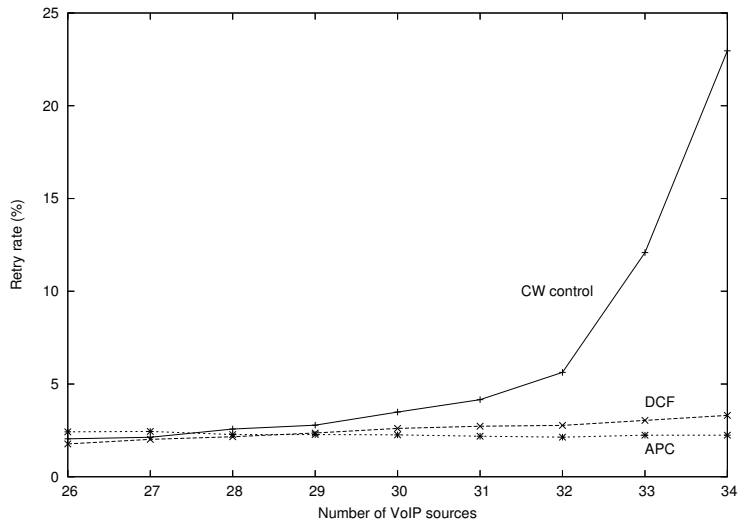

Fig. 11. Retry rate of the AP (downlink traffic) in three approaches: Controlling CW, DCF and APC

AP computes the average queue size of the wireless nodes with that information. However, this method necessitates changes not only in the AP but also in the clients. Another way to implement APC without changing the client is to estimate the queue size of the nodes. The queue size of the nodes is the number of packets generated in the application layer minus the number of packets sent to the AP. The AP can compute the number of packets sent to itself. We can calculate the number of the packets generated at the wireless nodes if we know the number of active wireless nodes, by dividing it by the packetization interval, and we can estimate the number of active wireless nodes by checking the received packets from wireless nodes. For example, if 10 wireless nodes are sending VoIP packets with $0.02 \mathrm{~s}$ packetization interval, we can see that $500(=10 / 0.02)$ packets are generated from all wireless nodes every second. The equation to estimate the average queue size of wireless nodes can be summarized as follows:

$$
Q_{i}=Q_{i-1}+\frac{\sum_{j=1}^{N_{i}}\left(\frac{t_{s}}{P I_{j}}-R_{j}\right)}{N_{i}}
$$

where, $Q_{i}$ is the estimated average queue size of wireless nodes at $i$ th sampling time, $N_{i}$ is the number of active wireless nodes at $i$ th sampling time, $t_{s}$ is sampling interval in milliseconds, $P I_{j}$ is the packetization interval of the wireless nodes $j$ in milliseconds, and $R_{j}$ is the number of packets the AP received from the wireless node $j$.

We have implemented this algorithm to estimate the queue size of wireless nodes, and integrated it into APC. Fig. 12 shows the simulation results of APC with the estimated queue size. Comparing this with Fig. 6(b), we can see that there is no difference between the results, thus confirming that the estimated queue size can be used instead of the actual queue size of wireless nodes to implement APC.

Another issue is to implement the contention free transmission at the MAC layer. Contention free transmission is easily implemented using IEEE 802.11e TXOP (Transmission Opportunity). In IEEE 802.11e, TXOP is defined as an interval of time when a station has the right to initiate the transmission, 


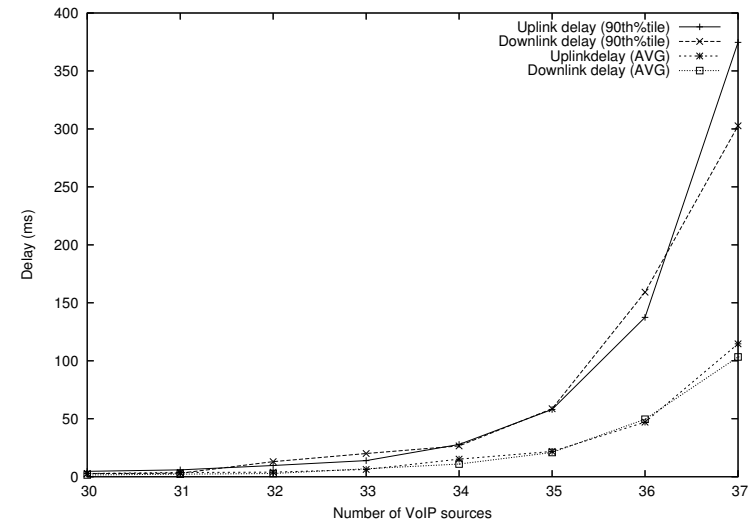

Fig. 12. Simulation results of the AP with the estimated queue size of nodes

and represented as start time and maximum duration, which are typically fixed according to the Access Category (AC). In APC, TXOP value needs to be changed dynamically according to the number of packets transmitted contention free (or the priority of the AP). Furthermore, WiFi alliance has developed Wireless Media Extension (WME), which is a subset of 802.11e feature including TXOP, as an interim solution for QoS of IEEE 802.11, and many manufacturers including Atheros have included the WME feature to their wireless cards. We have also confirmed that the TXOP value can be changed through a slight change in the wireless card driver.

\section{CONCLUSION AND FUTURE WORK}

We have shown that as the number of VoIP sources increases, the downlink delay increases significantly while the uplink delay remains low in DCF. This is because every wireless node including the AP has the same chance to transmit frames in DCF, while the AP needs to transmit more packets than wireless nodes. In this paper, we have proposed APC, which differentiates the priority of the AP from the wireless nodes adaptively according to the traffic volume and balances the uplink and downlink delay, by allowing the AP to transmit $Q_{A P} / Q_{N o d e}$ packets contention free. We have also analyzed the performance of APC theoretically and have proved that APC balances the uplink and downlink delay.

We have implemented the APC algorithm using the QualNet simulator and have shown that APC balances the uplink and downlink delay effectively in VoIP traffic with various packetization intervals.

We believe that APC can be implemented using IEEE 802.11e, and in the future, we will implement the APC using WME feature and compare the results with our simulation results. Also, we will evaluate the performance of APC with various background traffic such as HTTP or P2P.

\section{REFERENCES}

[1] D. J. Deng, R. S. Chang, and A. Veres, "A priority scheme for IEEE 802.11 DCF access method," IEICE Trans. Commun., vol. E82-B, no. 1, pp. $96-102$, Oct 1999.
[2] M. Barry, A. T. Campbell, and A. Veres, "Distributed control algorithms for service differentiation in wirless packet networks," in IEEE INFOCOM, Apr 2001, pp. 582-590.

[3] I. Aad and C. Castelluccia, "Differentiation mechanism for IEEE 802.11," in IEEE INFOCOM, Apr 2001, pp. 209-218.

[4] IEEE Draft Std. 802.11e, Medium Access Control (MAC) Enhancements for Quality of Service (QoS), D8.0 ed., IEEE, Feb 2004.

[5] S. Pilosof, R. Ramjee, D. Raz, Y. Shavitt, and P. Sinha, "Understanding TCP fairness over wireless LAN," in IEEE INFOCOM, Mar 2003, pp. 863-872.

[6] S. W. Kim, B.-S. Kim, and Y. Fang, "Downlink and uplink resource allocation in IEEE 802.11 wireless LANs," IEEE Trans. on Vehicular Technology, vol. 54, no. 1, pp. 320-327, Jan. 2005.

[7] J. Jeong, S. Choi, and C. kwon Kim, "Achieving weighted fairness between uplink and downlink in IEEE 802.11 DCF-based WLANs," in Qshine, August 2005.

[8] R. Jain, D. Chiu, and W. Hawe, "A quantative measure of fairness and discrimination for resource allocation in shared computer systems," DEC, Tech. Rep. TR-301, 1984.

[9] X. G. Wang, G. Min, Mellor, and J.E., "Improving VoIP application's performance over WLAN using a new distributed fair MAC scheme," in Advanced Information Networking and Applications, 2004. AINA 2004. 18th International Conference, vol. 1, 2004, pp. 126-131.

[10] Casetti, C. Chiasserini, and C.-F., "Improving fairness and throughput for voice traffic in 802.11e EDCA," in Personal, Indoor and Mobile Radio Communications, 2004. PIMRC 2004. 15th IEEE International Symposium, vol. 1, 2004, pp. 525-530.

[11] O.Tickoo and B.Sikdar, "Queueing analysis and delay mitigation in IEEE 802.11 random access MAC based wireless networks," in INFO. COM, 2004

[12] Wireless LAN Medium Access Control (MAC) and Physical (PHY) specifications:Higher-Speed Physical Layer Extention in the $2.4 \mathrm{GHz}$ Band, IEEE, 1999.

[13] QualNet Network Simulator. [Online]. Available: http://www.qualnet.com

[14] Wireless LAN Medium Access Control (MAC) and Physical (PHY) specifications, IEEE, 1999.

[15] H. Schulzrinne, S. Casner, R. Frederick, and V. Jacobson, RTP: A Transport Protocol for Real-Time Applications, Jul 2003.

[16] Artificial Conversational Speech, ITU-T P.59, 1993

[17] One-way Transmission Time, ITU-T G.114, 2003.

[18] Voice Quality Recommendations for IP Telephony, TIA, 2003. 